\title{
Phase-field model for activated reaction fronts
}

\author{
Nikolas Provatas \\ Research Institute for Theoretical Physics, University of Helsinki, P. O. Box 9, Siltavuorenpenger 20 C, FIN-00014 Helsinki, Finland \\ Martin Grant \\ Physics Department, McGill University, Rutherford Building, 3600 rue University, Montréal, Québec, Canada H3A 2T8 \\ K. R. Elder \\ Department of Physics and Materials Research Laboratory, University of Illinois at Urbana-Champaign, 1110 West Green Street, \\ Urbana, Illinois 61801 \\ (Received 3 August 1995)
}

\begin{abstract}
We propose a phase-field model to describe reaction front propagation in activated transitions obeying Arrhenius kinetics. The model is applicable, for example, to the explosive crystallization of amorphous films. Two coupled fields interact during the reaction, a temperature field $T(x, t)$ and a field $C(x, t)$ describing the amorphous/crystal transition, which are continuous functions of space $x$ and time $t$. Unlike previous work, our model incorporates a nonzero front width $\epsilon$ in a natural way, corresponding to that region in space where $T$ and $C$ undergo rapid variation. In the limit of $\epsilon \rightarrow 0$, our model reduces to the sharp interface approach of others. Treating the background temperature of the reacting sample as a control parameter, periodic solutions in $C$ and $T$ can be found which go through a series of period doubling bifurcations. We find that the substrate temperature marking the onset of period doubling bifurcations decreases with increasing concentration diffusion. Furthermore, it is shown that period doubling bifurcations of $C-T$ solutions of period greater than 2 are generated by dynamics isomorphic to those of the one-dimensional logistic map, for all values of concentration diffusion studied.
\end{abstract}

\section{INTRODUCTION}

Materials existing in a metastable state can undergo a transition into an energetically more favorable phase by chemical activation. In such processes, the metastable phase must overcome an energy barrier. For an amorphous material, this energy can correspond to that required for the glass to locally diffuse and reorient itself to attain a stable crystalline phase. The rate at which such a process occurs in a steady state is often well described by the Arrhenius law of chemical kinetics, $e^{-E / k_{B} T}$, where $E$ is the activation energy, $k_{B}$ is Boltzmann's constant, and $T$ is temperature. Such a reaction can start due to spontaneous fluctuations in the metastable phase, or, as in the case we shall consider, when the metastable phase is brought into contact with a more stable phase. A reaction front then forms between the two phases, which advances from the stable phase through the metastable phase, until no metastable phase remains. In exothermic reactions, latent heat is released at this front. Since the Arrhenius rate increases with temperature, such an exothermic reaction can lead to an increase in the reaction rate, causing the transition to develop a rapid, self-sustaining reaction front. In the case where the latent heat is lower than the activation energy, the metastable material can be elevated to some fixed temperature, thus still allowing for the formation of a front.

An experimental realization of such a reaction is found in the crystallization of amorphous films. The lower free energy of the crystalline state leads to the release of latent heat and initiates the formation of a rapid reaction front as discussed above. This scenario is known as explosive crystallization since the propagation velocities of the front can be meters per second. ${ }^{1-3}$ In these experiments, the amorphous film is on a substrate fixed at a temperature $T_{\text {sub }}$. It is $T_{\text {sub }}$ that determines whether or not the latent heat released will be great enough to maintain self-sustained crystallization. Below a critical value of $T_{\text {sub }}$, the latent heat released is insufficient to crystallize any amorphous material. The heat is thus lost to the substrate and the crystallization front stops. A striking feature of the process is that, for temperatures slightly above the critical substrate temperature, regular periodic variations in the film thickness, grain size, and completeness of transformation have been observed after crystallization in samples such as InSb and GaSb. ${ }^{1,4}$ Far away from nucleation centers, these surface undulations resemble parallel wave fronts, perpendicular to the direction of propagation of the crystallization front. Their periodic spacing reflects the fact that the temperature at the front varies periodically. The oscillating interfacial temperature leads to different local reaction rates and thus different local crystal densities, which can be measured optically. ${ }^{5}$ Similar periodic patterns have also been observed in oscillatory zoning in the solidification of binary mixtures, such as plagioclase feldspars, ${ }^{6}$ as well as other nonlinear systems. ${ }^{7,8}$

In the case of explosive crystallization, the origin of these undulations has been explained by van Saarloos, Weeks, and Kurtze, ${ }^{9}$ using the following sharp interface model for the temperature field. In such a model the amorphous/crystal transition is assumed to take place over a spatial region that is infinitely sharp. In this paper we have extended their analysis by including the coupling to a field $C(x, t)$ describing the amorphous/crystal transition. In this approach, 
$C(x, t)$ can vary independently and is not slaved to $T$ as in the sharp interface approach. The coupling between $C$ and $T$ will become important as the system gets deeper and deeper into the period doubling sequence where small perturbations can initiate large changes. It is in this regime that the internal structure of the front (and any time lag between $C$ and $T$ ) will play a role.

Hence, we propose a phase-field reaction-diffusion model describing the transformation of a metastable material to a stable one for chemically activated reactions limited by Arrhenius kinetics. We introduce a two-field model which couples the local concentration of reactant $C(x, t)$ as a function of position $x$ and time $t$ to the local temperature $T(x, t)$. The model describes the reaction and diffusion of reactant concentration in both the metastable and stable phases, as well as the dynamics of the accompanying thermal field. The model is examined in the context of reaction-front propagation and bifurcations in reaction-front velocities. In particular, adjusting a parameter controlling the background temperature $T_{\text {sub }}$ can cause the propagation of the temperature and concentration fields to undergo a sequence of period doubling bifurcations. As our model is aimed at a general description of reaction fronts in reaction-diffusion systems governed by Arrhenius activation, it provides a generalization of previous sharp interface models of explosive crystallization of amorphous materials. For crystallization, the field $C(x, t)$ would represent a Fourier component of the density field, which has negligible amplitude in the amorphous phase and large amplitude in the crystal phase. This association connects explosive crystallization to the larger body of reaction-diffusion theory already used to model other physical and chemical phenomena from first principles. ${ }^{10}$

A further aspect of our model is reactant diffusion, which allows us to study certain material-dependent properties of reaction-front propagation. In particular, the bifurcation structure of the solutions of our model changes. For example, the background temperature $T_{\text {sub }}$ at which periodic solutions first emerge varies with reactant diffusion. Indeed, in explosive crystallization, it has been experimentally established that the critical substrate temperature is material dependent. ${ }^{1,4,11-14}$ We furthermore find that the entire range of substrate temperatures over which oscillatory solutions occur is also strongly dependent on diffusion.

The outline of this paper is as follows. ${ }^{15}$ We introduce our model in Sec. II, and give an asymptotic analysis of it in Sec. III. This shows that our model reduces to a sharp interface model, in particular limits. In Sec. IV, we examine our model equations numerically. The bifurcation structure of their solutions is studied, with emphasis on how the bifurcation structure changes as a function of diffusion of the local reactant concentration. We furthermore make an analysis of the equations in the parameter range corresponding to our numerical work, providing support for some of the numerical results. Finally, in Sec. V we summarize our results.

\section{MODEL}

A description of an activated transition must account for spatial variations in local concentration, as well as, in the case of the formation of a reaction front, the emergence of a boundary layer of extent $\epsilon$ defining the zone separating the stable and metastable phases. Thus, we introduce a field $C(x, t)$ describing the local concentration of reacting (metastable) material, where $C=1$ in the completely unreacted phase of the sample, while in the reacted (stable) phase $C=0$. For explosive crystallization of amorphous films, the reacting phase is amorphous while the reacted phase corresponds to crystal. Across the interfacial boundary layer where the transition takes place, $0<C<1$. The scale over which the concentration varies from $C=0$ to $C=1$ is determined by the interfacial width $\epsilon$. We furthermore introduce a local temperature field $T$. It is is driven by the latent heat released by changes in concentration of the metastable phase. In our model, the dynamics of the temperature and concentration fields are described by

$$
\frac{\partial C}{\partial t}=D_{c} \nabla^{2} C-\gamma R(T) C
$$

and

$$
\frac{\partial T}{\partial t}=D_{T} \nabla^{2} T-\Gamma\left(T-T_{\mathrm{sub}}\right)-q \frac{\partial C}{\partial t} .
$$

In Eq. (1) the first term describes local reactant concentration diffusion in the system. The concentration diffusion constant is given by $D_{C}$ and, for simplicity, is taken as constant in both phases. The second term describes the rate of transformation of the metastable phase to the stable one due to chemical activation, where $\gamma$ is a constant. The rate of activation is taken to follow Arrhenius kinetics

$$
R(T)=Q e^{-E /\left(k_{B} T\right)}
$$

where $E$ is the activation energy of the transition, $k_{B}$ the Boltzmann factor, and $Q$ is a constant. In the case of explosive crystallization, the amorphous to crystal reaction rate is given by

$$
R(T)=Q e^{-E /\left(k_{B} T\right)}\left[1-e^{\left(L / k_{B}\right)\left(1 / T-1 / T_{C}\right)}\right] .
$$

This is typical for the reaction rate of a crystal from a melt ${ }^{16}$ as well as that describing polymorphic crystallization of oxide glasses. ${ }^{17}$ Here, the constants $T_{c}$ and $L$ are the melting temperature and latent heat of melting, respectively. The Arrhenius kinetics discussed in this paper are controlled by the left-hand side of Eq. (4), where it essentially takes the form of Eq. (3). Finally, the $R(T) C$ term in Eq. (1) ensures that the rate of the transition is proportional to the concentration of metastable material.

Equation (2) describes the evolution of the thermal field. The first term describes thermal diffusion, where the thermal diffusion constant is given by $D_{T}$. The heat source is given by $q \partial C / \partial t$ and replaces the term $q \delta\left(x-x_{b}(t)\right)$ typical in sharp interface models such as that of Gilmer and Leamy. ${ }^{18}$ To satisfy heat conservation we must have $q \approx L / C_{p}$ where $L$ is the latent heat of the reaction and $C_{p}$ the specific heat, which is again taken to be the same in both phases. This source term generates latent heat over the interfacial length $\epsilon$ of the interface. The term $\Gamma\left(T-T_{\text {sub }}\right)$ is introduced to approximately describe heat loss through thermal dissipation by Newton's law of cooling. The background to which heat is dissipated, e.g., an underlying substrate, is held fixed at a 
constant temperature $T_{\text {sub }}$. In amorphous crystallization, this corresponds to a substrate on which a thin amorphous film rests.

\section{SHARP INTERFACE LIMIT}

It is instructive to study the limit in which Eqs. (1) and (2) simplify to a sharp interface model analogous to those used in Ref. 9 to model explosive crystallization (an equation for the thermal field plus a moving boundary condition). With the transformation to the dimensionless variables $x \rightarrow\left(\Gamma / D_{T}\right)^{1 / 2} x, \quad t \rightarrow \Gamma t$, and $T \rightarrow T / q$, this gives a model with three free parameters: $\rho=D_{C} / D_{T}, g=\gamma / \Gamma$, and $T_{0}=T_{\text {sub }} / q$. We solve our model in one dimension, simulating front propagation far from the nucleation site. The $C-T$ fronts satisfy the following conditions: $C(\infty, t)=1$, $C(-\infty, t)=0$, and $T( \pm \infty, t)=T_{0}$. Also, for $x \leqslant x\left(T=T_{m}\right)$, where $T_{m}$ is the maximum temperature along $x$ at a given time, $C(x, t) \approx 0$. We rewrite Eqs. (1) and (2), in a reference frame whose origin is at the maximum of the $T$ front. In this reference frame the dimensionless model of Eqs. (1) and (2) becomes

$$
\frac{\partial C}{\partial t}=\rho \frac{\partial^{2} C}{\partial x^{2}}+V(t) \frac{\partial C}{\partial x}-g R(T) C
$$

and

$$
\frac{\partial T}{\partial t}=\frac{\partial^{2} T}{\partial x^{2}}+V(t) \frac{\partial T}{\partial x}-\left(T-T_{0}\right)-S(x, t),
$$

where the source term in Eq. (36) is just

$$
S(x, t)=\frac{\partial C}{\partial t}-V(t) \frac{\partial C}{\partial x} .
$$

We will now consider the limit where variations of $x$ happen over a small length scale $\xi \rightarrow 0$. We identify $\xi$ with the term in the concentration field with the highest derivative ${ }^{19,20}$ so that $\xi=\sqrt{\rho / g}$. To consistently order the other terms, we let $1 / g=A \xi^{\nu}$, where $0<\nu<1$, and $A$ is a constant of order 1 .

If $x<|\epsilon|$ is the width of the boundary layer of the $C$ field, we can consider the two cases of the outer region given approximately by $x>|\epsilon|$, and the inner interface region given by $x<|\epsilon|$. The size of the inner region will be determined self-consistently to be $|\epsilon| \propto \xi^{\nu}$. On the outer domain we define an expansion of the $C$ and $T$ fields denoted by

$$
C_{\text {out }}=C_{\text {out }}^{0}(x, t)+\xi^{\nu} C_{\text {out }}^{1}(x, t)+\cdots
$$

and

$$
T_{\text {out }}=T_{\text {out }}^{0}(x, t)+\xi^{\nu} T_{\text {out }}^{1}(x, t)+\cdots,
$$

along with the expansion for the velocity, given by $V(t)=V^{0}(t)+\xi V^{1}(t)+\cdots$. The outer expansions must satisfy

$$
A \xi^{\nu} \frac{\partial C_{\text {out }}}{\partial t}=\xi^{2} \frac{\partial^{2} C_{\text {out }}}{\partial x^{2}}+A \xi^{\nu} V(t) \frac{\partial C_{\text {out }}}{\partial x}-R\left(T_{\text {out }}\right) C_{\text {out }}
$$

and

$$
\frac{\partial T_{\text {out }}}{\partial t}=\frac{\partial^{2} T_{\text {out }}}{\partial x^{2}}+V(t) \frac{\partial T_{\text {out }}}{\partial x}-\left(T_{\text {out }}-T_{0}\right)-S_{\text {out }},
$$

where the source term in Eq. (11) is just

$$
S_{\text {out }}(x, t)=\frac{\partial C_{\text {out }}}{\partial t}-V(t) \frac{\partial C_{\text {out }}}{\partial x} .
$$

The inner domain is examined by introducing the coordinate stretching $x=z \xi^{\nu}$, which transforms Eqs. (5) and (6) into

$A \xi^{\nu} \frac{\partial C_{\mathrm{in}}}{\partial t}=\xi^{2(1-\nu)} \frac{\partial^{2} C_{\mathrm{in}}}{\partial z^{2}}+A V(t) \frac{\partial C_{\mathrm{in}}}{\partial z}-R\left(T_{\mathrm{in}}\right) C_{\mathrm{in}}$

and

$\xi^{2 \nu} \frac{\partial T_{\text {in }}}{\partial t}=\frac{\partial^{2} T_{\text {in }}}{\partial z^{2}}+\xi^{\nu} V(t) \frac{\partial T_{\text {in }}}{\partial z}-\xi^{2 \nu}\left(T_{\text {in }}-T_{0}\right)-S_{\text {in }}$

where the source term in Eq. (14) is now

$$
S_{\text {in }}(z, t)=\xi^{2 \nu} \frac{\partial C_{\text {in }}}{\partial t}-\xi^{\nu} V(t) \frac{\partial C_{\text {in }}}{\partial z}
$$

and the inner expansions $C_{\mathrm{in}}$ and $T_{\mathrm{in}}$ are defined by

$$
C_{\mathrm{in}}=C_{\mathrm{in}}^{0}(z, t)+\xi^{\nu} C_{\mathrm{in}}^{1}(z, t)+\cdots
$$

and

$$
T_{\text {in }}=T_{\text {in }}^{0}(z, t)+\xi^{\nu} T_{\text {in }}^{1}(z, t)+\cdots
$$

We deal with the lowest order outer expansions by substituting the outer expansions into Eq. (10) and (11) and obtain, to lowest order, that $R\left(T_{\text {out }}^{0}\right) C_{\text {out }}^{0}=0$ where $C_{\text {out }}^{0}(-\infty, t)=0$ and $C_{\text {out }}^{0}(\infty, t)=1$. This implies that in the outer domain

$$
C_{\text {out }}^{0}=\Theta(x)
$$

where $\Theta(x)$ is the step function, and $R\left(T_{\text {out }}^{0}\right)=0$ for $x>0$. As a consequence, for $|x|>0, T_{\text {out }}^{0}$ satisfies

$$
\frac{\partial T_{\mathrm{out}}^{0}}{\partial t}=\frac{\partial^{2} T_{\mathrm{out}}^{0}}{\partial x^{2}}+V^{0}(t) \frac{\partial T_{\mathrm{out}}^{0}}{\partial x}-\left(T_{\mathrm{out}}^{0}-T_{0}\right) .
$$

Considering the temperature field in the inner domain, $T_{\text {in }}^{0}$ satisfies $d^{2} T_{\text {in }}^{0} / d z^{2}=0$, whose solution is $T_{\text {in }}^{0}=a z+b$. Matching $T_{\text {in }}^{0}$ to $T_{\text {out }}^{0}$ using Van Dyke's matching principle, ${ }^{19,20}$ it is straightforward to show that

$$
T_{\mathrm{in}}^{0}(z, t)=T_{\mathrm{out}}^{0}(0, t) \text {. }
$$

Thus as $\xi \rightarrow 0$ the inner temperature is, to lowest order, the $x \rightarrow 0$ limit of the outer temperature. Going to order $\xi^{\nu}$ in Eq. (14), we obtain

$$
\frac{\partial^{2} T_{\text {in }}^{1}}{\partial z^{2}}=-V^{0} \frac{\partial C_{\text {in }}^{0}}{\partial z} .
$$

Integrating Eq. (21) yields a two-term expansion of $T_{\text {in }}$. Matching this two-term (order $\xi^{\nu}$ ) expansion of $T_{\text {in }}$ with the one-term outer expansion $T_{\text {out }}^{0}$ of Eq. (19), using Van Dyke's rule, ${ }^{20,19}$ gives 


$$
\frac{d T_{\mathrm{out}}^{0}\left(x \rightarrow 0^{+}, t\right)}{d x}-\frac{d T_{\mathrm{out}}^{0}\left(x \rightarrow 0^{-}, t\right)}{d x}=-V^{0}(t) .
$$

Equation (22) is the latent heat condition encountered in sharp interface models. Equations (19) and (22) are arrived at in a manner analogous to that in which Stefan and HeleShaw models are derived as asymptotic limits of phase-field equations of model $C .{ }^{21}$ From Eq. (21) it can also be shown that $T_{\text {in }}(z)$ drops off over a range of order $x \sim\left[d T_{\text {out }}^{0}\left(0^{+}, t\right) / d x\right]^{-1}$. This therefore defines the order of the range of validity of Eq. (20). But, since $z=x / \xi^{\nu}$ is of order 1, the width of the interface satisfies $|\epsilon| \sim O\left(\xi^{\nu}\right)$.

To lowest order, Eq. (13) leads to an equation for $C_{\text {in }}^{0}$ given by

$$
A V^{0}(t) \frac{\partial C_{\mathrm{in}}^{0}}{\partial z}-R\left(T_{\mathrm{in}}\right) C_{\mathrm{in}}^{0}=0 .
$$

Since this is linear, it can be integrated to give

$$
C_{\mathrm{in}}^{0}(z, t)=\exp \left(-\int_{z}^{\infty} \frac{R\left[T_{\mathrm{in}}(y, t)\right]}{A V^{0}(t)} d y\right) .
$$

Furthermore, since $T_{\text {in }}(z) \rightarrow T_{0}$ (the background temperature) as $|z| \rightarrow \infty$, Eq. (24) automatically satisfies the required boundary conditions $C_{\text {in }}^{0}(z \rightarrow-\infty, t)=0$ and $C_{\text {in }}^{0}(z \rightarrow \infty, t)$ $=1$. With respect to the outer variable, $x=z \xi^{\nu}$, we see that

$$
\lim _{\xi \rightarrow 0} \frac{d C_{\mathrm{in}}^{0}(z)}{d x}=\lim _{\xi \rightarrow 0} \frac{1}{\xi^{\nu}} \frac{d C_{\mathrm{in}}^{0}(z)}{d z} \rightarrow \delta(x) .
$$

Equations (19), (22), and (25) can be combined into one equation describing the temperature field to lowest order. This is given by

$$
\frac{\partial T_{\text {out }}^{0}}{\partial t}=\frac{\partial^{2} T_{\text {out }}^{0}}{\partial x^{2}}+V^{0} \frac{\partial T_{\text {out }}^{0}}{\partial x}-\left(T_{\text {out }}^{0}-T_{0}\right)+V^{0} \delta(x) .
$$

Equation (26) is precisely the form used to describe the temperature field by sharp interface models. All that is required to make it self-consistent is a solvability condition between $V^{0}(t)$ and $T_{\text {out }}^{0}(0, t)$.

To obtain a solvability condition, we begin by noting that substitution of Eq. (20) into Eq. (24) gives a solution that diverges as $z \rightarrow \infty$. From our discussion following Eq. (22), there must exist a $z=z_{p} \sim O(1)$ such that for $-\infty<z<z_{p}$ one can replace $T_{\text {in }}$ in Eq. (24) by Eq. (20). On this interval $C_{\text {in }}^{0}$ becomes

$$
C_{\mathrm{in}}^{0}=F \exp \left(\frac{R\left[T_{\mathrm{out}}^{0}(0, t)\right]}{A V^{0}(t)}\left(z-z_{p}\right)\right),
$$

where $F=\exp \left(-\int_{z_{p}}^{\infty} R\left[T_{\mathrm{in}}(y, t)\right] / A V^{0}(t) d y\right)$. The order of $F$ will be determined as we proceed. For $z>z_{p}$ we need all orders of $T_{\mathrm{in}}(z, t)$ to proceed further with Eq. (24). To find a form of $C_{\text {in }}^{0}$ on $z_{p}<z<\infty$, we note that in obtaining Eq. (23) the variable $z$ was chosen so as to balance the second and third terms of Eq. (13). It is also possible, however, to balance the first and second terms, obtaining a solution valid for $z>z_{p}$. Specifically, consider the transformation $z-z_{p}=\xi^{2(1-\nu)} \eta$. With this stretching equation, Eq. (13) gives

$$
\frac{\partial^{2} C_{\mathrm{in}}^{0}}{\partial \eta^{2}}+A V^{0} \frac{\partial C_{\mathrm{in}}^{0}}{\partial \eta}=0
$$

for $C_{\text {in }}^{0}$ on $z_{p}<z<\infty$, or $\eta>0$. Expanding $C_{\text {in }}^{0}$ as $C_{\text {in }}^{0}=1+\xi^{2(1-\nu)} \tilde{C}_{\text {in }}^{1}(\eta, t)+\cdots$, the solution for $\tilde{C}_{\text {in }}^{1}$ becomes

$$
\tilde{C}_{\text {in }}^{1}=-B \exp \left[-A V^{0}(t) \eta\right]
$$

with $B$ some positive constant. Expanding Eq. (29) to first order in $\eta$, near $\eta=0$, writing it in terms of the variable $z$, and then matching $C_{\text {in }}^{0}$ of Eq. (28) with the first order expansion of $C_{\text {in }}^{0}$ in Eq. (27), we arrive at the velocity condition

$$
V^{0}(t)=\sqrt{\frac{F}{B}} \frac{\tilde{R}\left[T_{\mathrm{out}}^{0}(0, t)\right]}{A},
$$

where $\tilde{R}=\sqrt{Q} \exp \left(-E / 2 k_{B} T\right)$ and $F=1-B \xi^{2(1-\nu)}$. The solvability condition imposed by Eq. (30) implies that, as $\xi \rightarrow 0$, the front velocity is an Arrhenius function of the peak front temperature. Up to a trivial factor of 2 in $E / k_{B} T$, this is the same result as for the previous sharp interface models of explosive crystallization. The choice of $z_{p}$ only changes the constants in the square root of Eq. (30) but not its form. Likewise, the magnitude of $F$ only differs from 1 by order $\xi^{2(1-\nu)}$.

\section{BIFURCATIONS IN FRONT PROPAGATION IN THE MODEL}

In this section the field equations, Eqs. (1) and (2), are solved numerically. Temperature is measured in units of $q$, distance in units of reactant (crystal grain) size, and time in units of seconds. Simulations were done using an explicit Euler time step algorithm with a spatial step of $d x=1$. The model was solved in one dimension to simulate the reaction front at late times, sufficiently far from the initiation site. We used $d t=0.013$, which is small enough that our results remain essentially unchanged by making $d t$ smaller. In units of $d t$, the maximum time studied was $t \sim 170000$, with our reported data based typically on the subsequent 1200 to 2400 time iterates. The dimensionless constants in Eq. (4) were taken as follows: $E /\left(q k_{B}\right)=12.952, T_{c}=1.8, L /\left(q k_{B}\right)=3$, while $Q=209165.328 \gamma$. These are the same as the constants used in Ref. 9 for the dimensionless reaction rate describing the amorphous to crystalline transition, where $Q$ and $E$ are adjusted so that a maximum dimensionless growth rate of 12 is attained at $T=1.6$. With $q \sim 300 \mathrm{~K}$, the values of $E, L$, and $T_{c}$ are also consistent with amorphous $\mathrm{Sb} .{ }^{9}$ For our numerical simulations we fixed $\gamma=1 \mathrm{~s}^{-1}, \Gamma=0.02 \mathrm{~s}^{-1}$, and $D_{T}=20 \mathrm{~s}^{-2}$ keeping $D_{C}$ and $T_{0}$ variable. For greater generality, we will present our results in terms of dimensionless variables.

In Figs. 1 and 2, the evolution of $C$ and $T(x, t)$ fronts is shown for three different values of the substrate temperature $T_{0}$ with $\rho=0.05$. Figure 1(a) shows a constant velocity propagation of the $T$ field for $T_{0}=0.323$. The horizontal axis is space and the vertical time. The gray scale represents tem- 


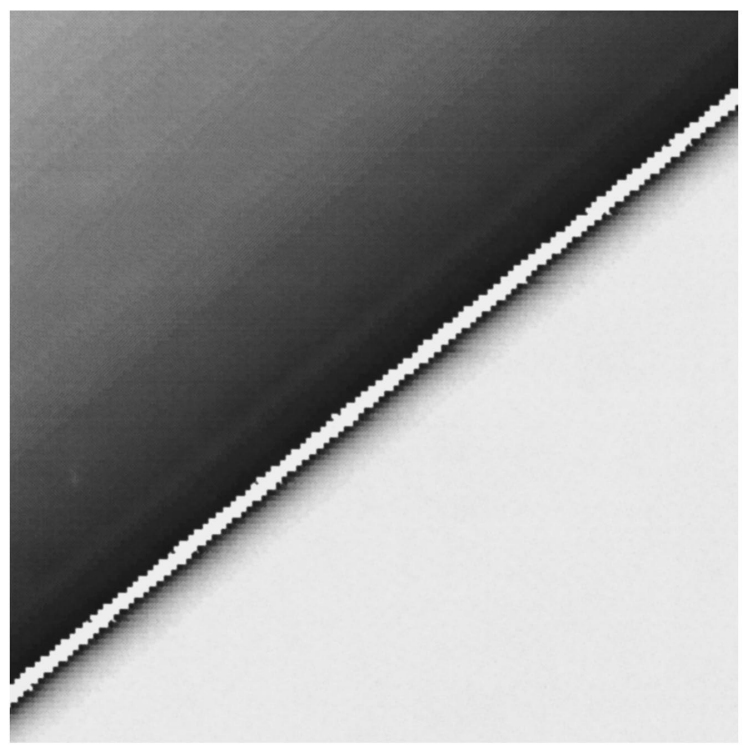

(a)

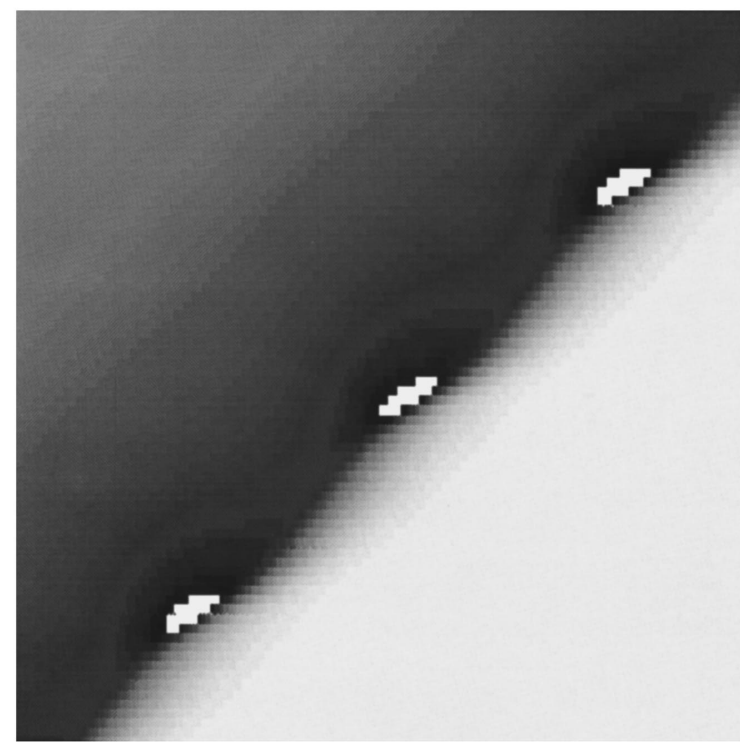

(b)

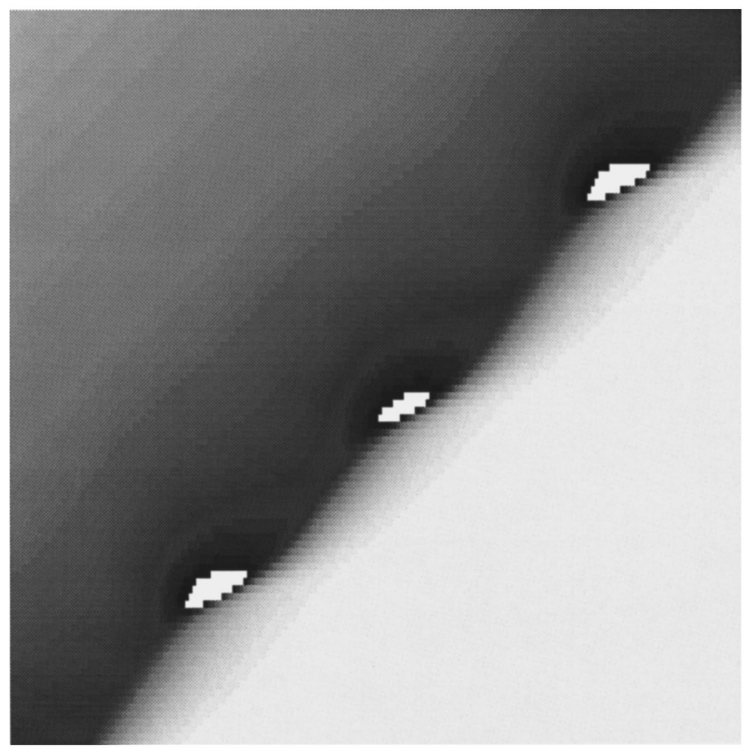

(c)

FIG. 1. Evolution of temperature field $T$ for three different $T_{0}$ with $\rho=0.05$. The horizontal axis represents space, the vertical axis is time, and the gray scale temperature. The darker the pixels the higher the temperature; the straight white band in the central dark regions represents the hottest part of the front. (a) $T_{0}=0.332$. Linear slope implies front is moving at constant speed. Scales: $2424 \leqslant x \leqslant 2675$, $1 \leqslant t \leqslant 130$ in time units of $50 d t$. (b) $T_{0}=0.328$. Periodic front motion is evident. Peak temperatures oscillate in period- 2 cycle. Periodicity apparent in the periodic spacing between successive temperature maxima. Scales: $2280 \leqslant x \leqslant 2455,30 \leqslant t \leqslant 107$. (c) $T_{0}=0.326$. Periodic front motion with peaks in $T$ every third peak. First white peak on lower left is the same as the last on the upper right. This is a period-4 solution. Close inspection reveals that spacing between the peaks in the $T$ field also repeats with a period- 4 cycle. Scales: $8055 \leqslant x \leqslant 8255$, $1 \leqslant t \leqslant 100$. Periodic solutions also evident in the oscillations of the diffusion tail behind the leading front.

perature. The darker the pixels the higher the temperature, while the lighter areas to the top left and bottom right of the figure represent cool areas. The straight white band running through the dark middle zone defines the maximum temperature front. The uniform propagation is marked by a straight line front. Figure 2(a) shows the propagation of the $C$ field corresponding to the $T_{0}$ of Fig. 1(a). The white area represents reacted material and the dark area, ahead of the interface, unreacted. In Fig. 1(b) $T_{0}=0.328$, and we now see the emergence of periodic solutions in the evolution of $T$. Peaks in temperature now occur at regular intervals in time, giving a period-2 solution. The locations of higher temperatures also correspond to faster propagation velocities, given by the slope of the front. Also, it is clear that the width of the interface $\epsilon$ also oscillates with a period-2 cycle. This is shown in Fig. 2(b), which corresponds to the $C$ field of Fig. 1(b). Decreasing $T_{0}$ to $T_{0}=0.326$ generates higher-order periodic solutions. Figure 1(c) and Fig. 2(c) show a periodic solution of the temperature and concentration fields that repeats with a period- 4 cycle: The peak temperatures repeat on 


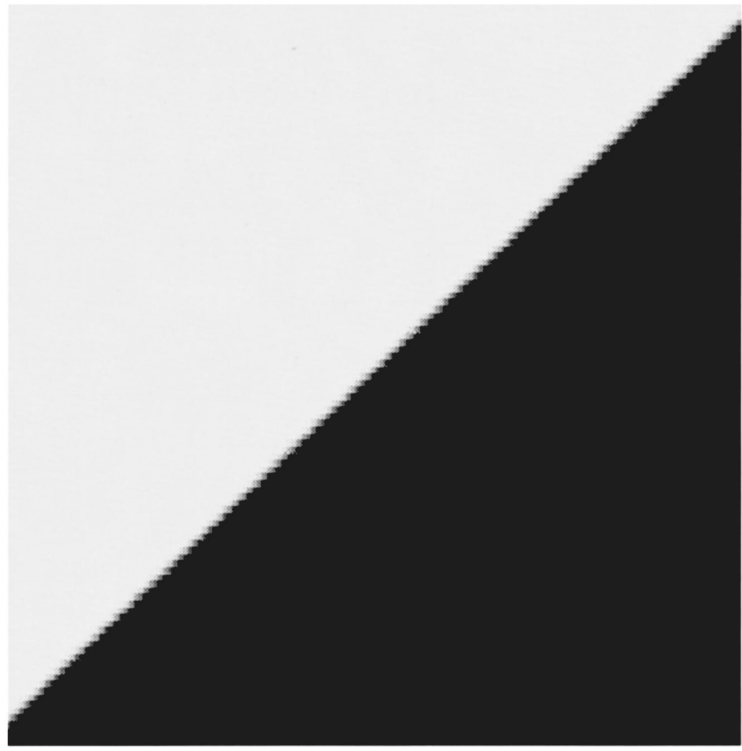

(a)

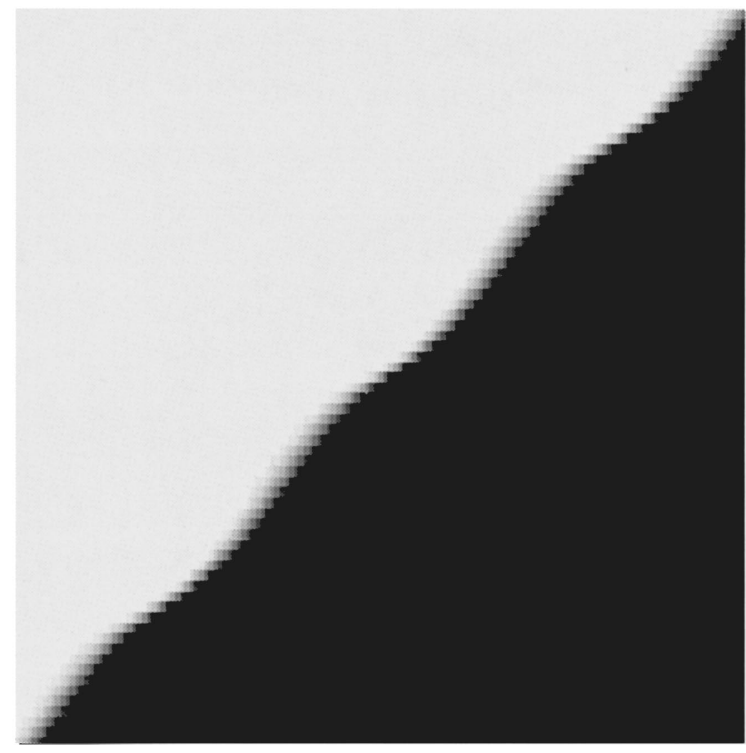

(b)

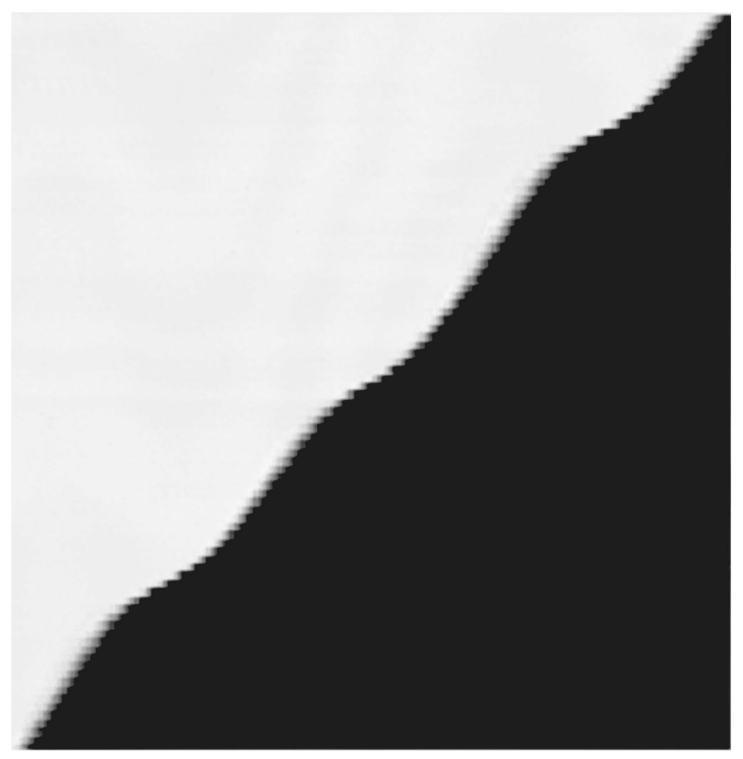

(c)

FIG. 2. Evolution of $C$ field corresponding to Fig. 1. White regions are reacted sample while the dark are unreacted phase. Fuzzy regions in between are due to the interface between the two phases. For (b) (period 2) and (c) (period 4), the periodicity is best seen in the period-2 or period-4 oscillations in the slope of the interface. In (a), the distance range is as in Fig. 1(a) while $5 \leqslant t \leqslant 115$. In (b), $2290 \leqslant x \leqslant 2445$, while $t=1$ to $t=90$. In (c), $8075 \leqslant x \leqslant 8255$ and $30<t<100$. Due to thermal diffusion, the $T$ field changes over a greater distance than the $C$ field and so the time and space ranges used to represent the $C$ fields are smaller than their counterparts for $T$.

every third peak, and the periodicity is evident in the slope of the $C$ interface.

A convenient way of studying the periodic evolution of the $C-T$ fields is to plot $T_{m}(t)$, the maximum temperature along $x$ at a given time. In Fig. 3 three solutions of $T_{m}(t)$, from bottom to top, are shown exhibiting solutions with period-2, -4 , and -8 cycles, respectively.

Plotting the extrema of $T_{m}(t)$, denoted $T_{E}$, as a function of the substrate temperature $T_{0}$ produces a bifurcation diagram of $T_{E}$. A bifurcation diagram displays the period and amplitudes that $T_{m}(t)$ attains, as a function of $T_{0}$. In Fig. 4, a bifurcation diagram of $T_{E}$ is shown for $\rho=0.125$. The inset of Fig. 4 shows an enlargement of the lower period-2 branch of the main figure. The inset within the bifurcation diagram is the sub-bifurcation diagram. The transition from one periodic solution of $T_{E}$ to another corresponds to period doubling; we will show that the sub-bifurcation diagram is generated by the same dynamics which generate period doubling in the one-dimensional (1D) logistic map. ${ }^{22}$ It is useful to define the values of $T_{0}$ where the various bifurcations of $T_{E}$ occur. The temperature $T_{0}(2)$ is the substrate temperature where $T_{E}$ bifurcates to period 2, and generally $T_{0}(p)$ denotes the value of $T_{0}$ where $T_{E}$ bifurcates to period $p$, with $p=2^{n}, n=1,2,3, \ldots$. Similarly, $T_{E}^{i}(p), i=1,2, \ldots, n$ denote the $n$ values of $T_{E}(p)$ (from bottom to top) at the bifurcation point $T_{0}(p)$ 


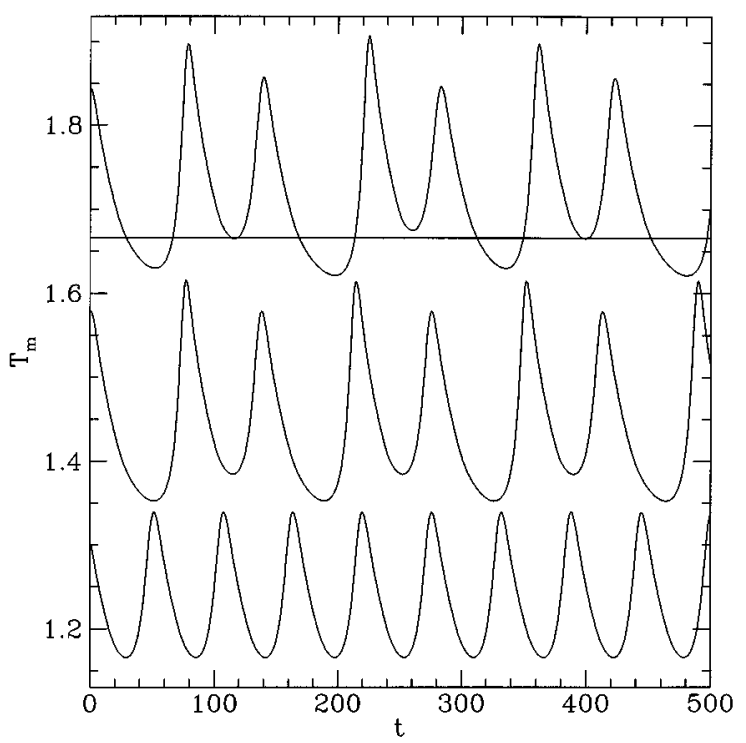

FIG. 3. Plots of $T_{m}(t)$ displaying period-2, 4 , and -8 solutions (bottom to top, respectively). We set $\rho=0.05$ and $T_{0}=0.328,0.326$, and 0.32587 , respectively. The curves have been translated so as not to overlap. The line through the top curve is drawn to emphasize the period-8 cycle of the trajectory.

Bifurcation diagrams were obtained for several values of $\rho$. Figure 5 shows the bifurcation diagrams for $\rho=0,0.025$, 0.05, 0.075, 0.1, 0.125, and 0.15. Inspection of Fig. 5 shows a change in the structure and location of the bifurcation diagrams as a function of $\rho$. Note also the increasing amplitude of higher periodic solutions, for larger $\rho$. These trends illustrated in Fig. 5 continue for $\rho>0.15$.

We refer to the $T_{0}$ shift caused by different $\rho$ as postponement. In Fig. $6, T_{0}(2)$ has been plotted as a function of $\rho$.

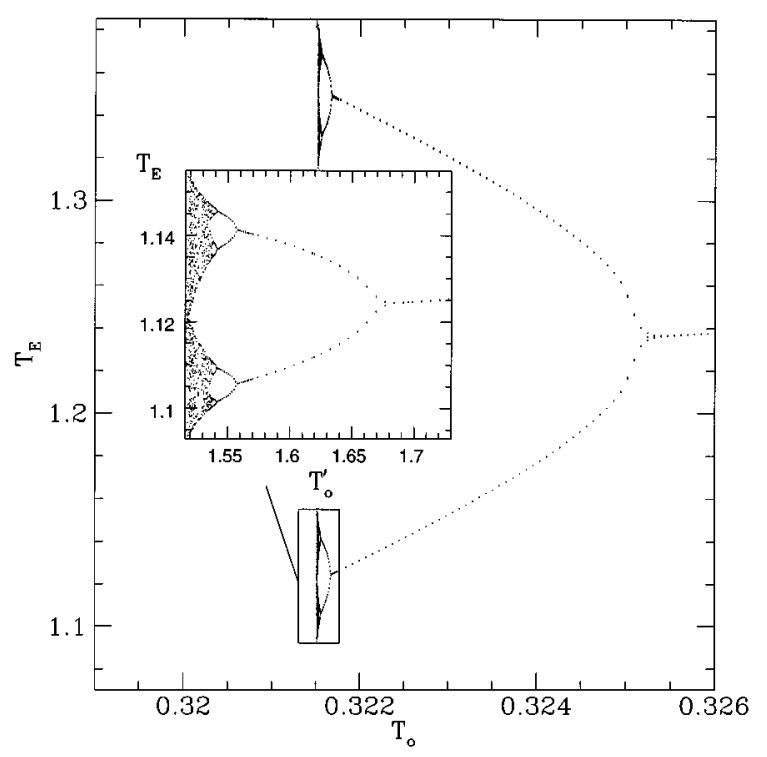

FIG. 4. A bifurcation diagram of the extremum of $T_{m}(t)$ for $\rho=0.125$. The inset shows an enlargement of the smaller subbifurcation diagram connected to the lower period-2 branch. Note the self-similarity to smaller and smaller scales. For clarity the $T_{0}$ axis of the inset has been rescaled by $T_{0}^{\prime}=\left(T_{0}-0.32\right) \times 1000$.

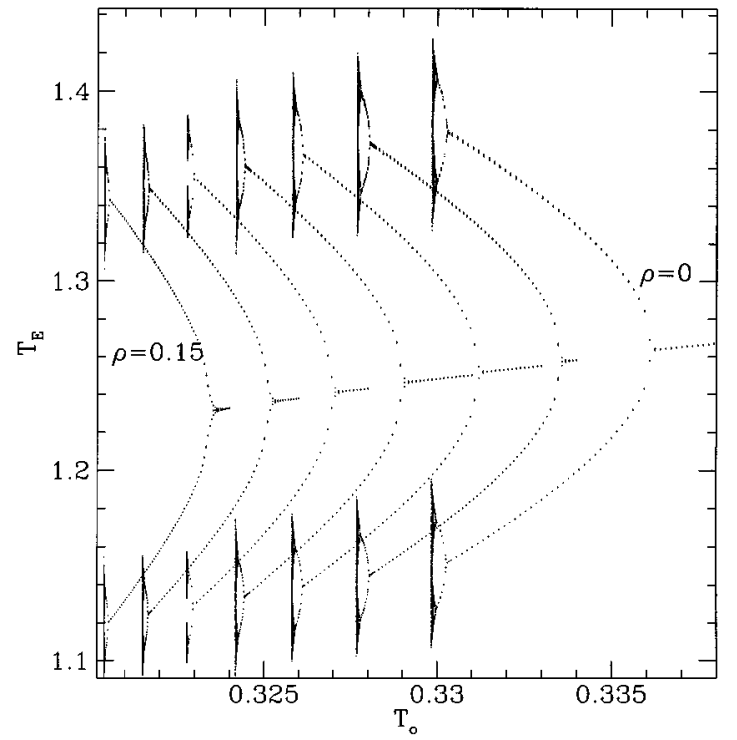

FIG. 5. Superposition of bifurcation diagrams for several values of $\rho$. As $\rho$ increases, the entire bifurcation diagram undergoes an overall translation as well as a contraction. This implies that the individual period doubling windows each contract. For the larger values of $\rho$, the higher period doubling windows cannot be resolved.

We find that $T_{0}(2)$ is fitted well by an exponential function of the form

$$
T_{0}(2)=\left.T_{0}(2)\right|_{\rho=0}+a\left(1-e^{-b \rho}\right),
$$

with $a \approx 0.306$ and $b \approx 3.6$. We will see below that this fit of $T_{0}(2)$ is also supported by analytic arguments. In explosive crystallization, the point $T_{0}(2)$ is experimentally the most easily accessible bifurcation point, as it is the substrate temperature at which periodic surface patterns first emerge in the crystallized sample. These patterns correspond to variations

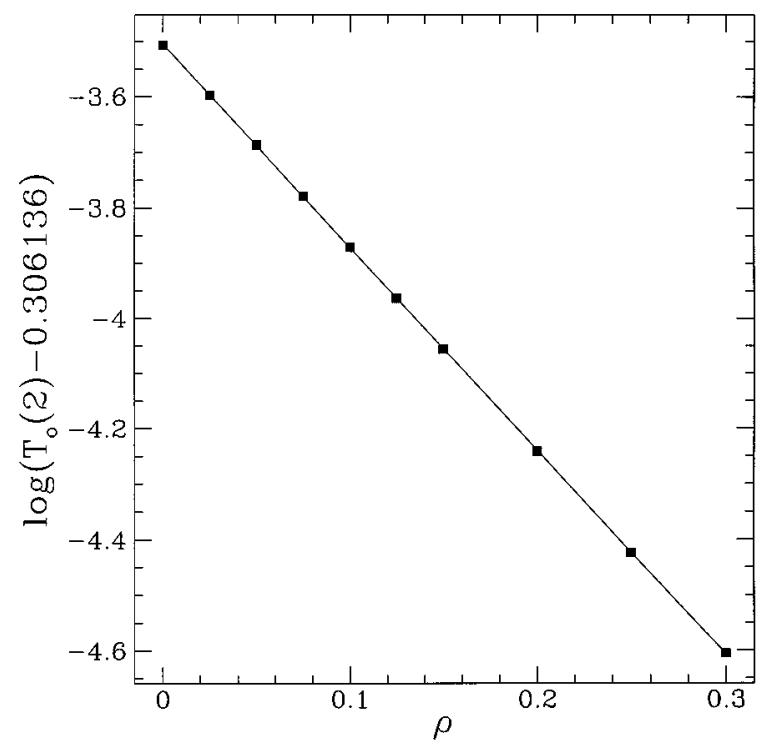

FIG. 6. Plot of natural logarithm $\ln \left[T_{0}(2)-0.306\right]$ versus $\rho$. $T_{0}(2)$ denotes the substrate temperature at which stable steady-state solutions bifurcate to period-2 solutions. 


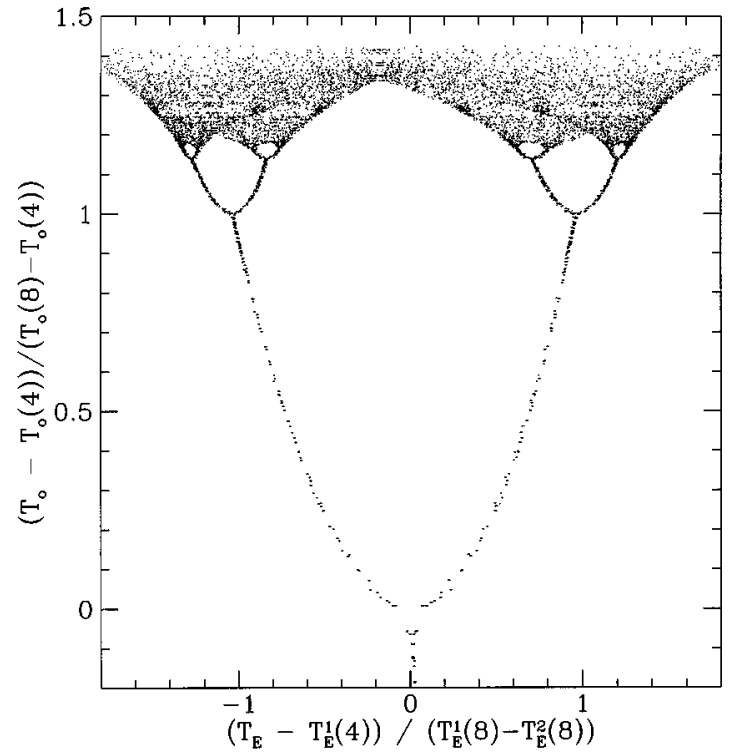

FIG. 7. Mapping onto one curve of all the sub-bifurcation diagrams attached to the lower period-2 branches of Fig. 5. The transformation that maps these sub-bifurcation diagrams onto a common curve does not also scale the larger period-2 sections onto a common curve.

in film thickness and grain size due to the periodically varying temperatures that sweep through the sample during the amorphous to crystal transformation. ${ }^{4}$ Their detection can be achieved by exploiting the optical properties of the amorphous and crystal phases of the thin film. ${ }^{5}$

There is a structural difference between the period-2 branches of $T_{E}$ bifurcation diagrams and branches of higher periods. This is best illustrated by rescaling the $\left(T_{0}, T_{E}\right)$ axes of Fig. 5 according to the linear transformation

$$
\tilde{T}_{0}=\frac{T_{0}-T_{0}(4)}{T_{0}(8)-T_{0}(4)}, \quad \tilde{T}_{E}=\frac{T_{E}-T_{E}^{1}(4)}{T_{E}^{1}(8)-T_{E}^{2}(8)} .
$$

This rescaling collapses all the lower sub-bifurcation diagrams of Fig. 5 onto a single bifurcation diagram. Figure 7 illustrates this collapse. There is a clear resemblance to the period doubling bifurcations generated by the logistic map. ${ }^{22}$ Applying Eq. (32) to the period-2 $\left(T_{0}, T_{E}\right)$ portion of Fig. 5 does not also collapse the period- 2 branches onto a common curve, indicating a difference between the period- 2 branches of the bifurcation diagrams and those of higher period. Changing the superscripts 1 and 2 in the denominator of the $T_{E}$ transformation similarly collapses all the upper subbifurcation diagrams onto the same common curve.

Defining the $T_{0}$ interval between period- $2^{n}$ and period$2^{n+1} \quad$ bifurcations as $W_{2^{n}}=T_{0}\left(2^{n}\right)-T_{0}\left(2^{n+1}\right)$ $(n=1,2,3, \ldots)$, Fig. 7 implies

$$
\frac{W_{2^{n}}}{W_{2^{n+1}}}=C_{n}
$$

for $n=2,3, \ldots$, and for all $\rho$. A plot of $W_{4}$ and $C_{2} W_{8}$ is shown in Fig. 8 for $n=2$, where $C_{2} \sim 6.978$. For $\rho \geqslant 0.2$ differences between $W_{4}$ and $W_{8}$ are of the same order as their absolute values, which reflects inaccuracies in bifurcation structures for $T_{0}<T_{0}(8)$ as $\rho$ increases. Indeed, finer struc-

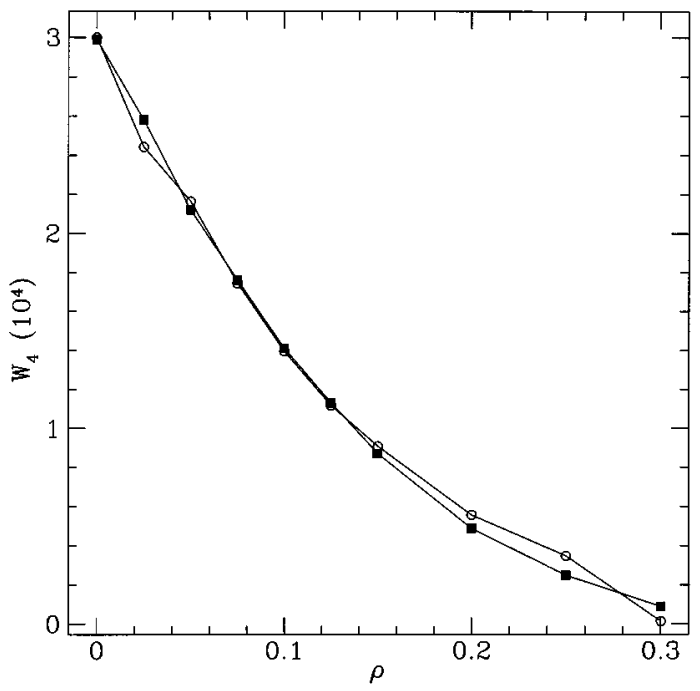

FIG. 8. Plot showing self-similarity of the period- 4 and period- 8 windows of Fig. 5. The square symbols represent $W_{4}$ and the circles $C_{2} W_{8}$. The constant $C_{2}$ is the scaling factor that scales $W_{8}$ to the size of $W_{4}$.

tures of the sub-bifurcation diagrams [for $\left.T_{0}<T_{0}(8)\right]$ will inevitably approach the same order as inaccuracies of our numerical methods. Furthermore, larger $\rho$ values require a greater transient time before a nonchanging bifurcation pattern is established. Thus while larger structures $\left[T_{0}>T_{0}(8)\right]$ do collapse onto the curve of Fig. 7, the finer structures do not. For this reason, we did not include $\rho \geqslant 0.2$ in Fig. 7 .

The dynamics leading to the bifurcation properties discussed above can be understood by projecting the dynamics of $T_{E}$ onto a first return map. This relates $T_{E}^{i+1}$ to $T_{E}^{i}$, where now the index $i$ denotes the $i$ th branch of $T_{E}$. Figure 9 shows this for $\rho=0$ and 0.125 . In both cases $T_{0}$ was set [via transformation Eq. (32)] to correspond to a very high (possibly chaotic) orbit. A sample trajectory of iterates defined by the $\rho=0$ map $T_{E}^{i+1}=f\left(T_{E}^{i}\right)$ is shown. The maps shown in Fig. 9 both possess a minimum component. For all $\rho$ examined, these minimum components of $T_{E}^{i+1}=f\left(T_{E}^{i}\right)$ can be rescaled onto a common curve that is fitted by a quadratic function. The transformations that perform the rescaling of the quadratic components do not also line up the linear segments of $T_{E}^{i+1}=f\left(T_{E}^{i}\right)$. Moreover, the iterates of $f^{4}, f^{8}$, $f^{16}, \ldots$ always lie on the quadratic section of $f$. Thus, for all $\rho$, the iterates $T_{E}^{2^{i}}$, for $i \geqslant 2$ [corresponding to solutions of period 4 or greater which occur for $\left.T_{0}<T_{0}(4)\right]$, are generated by the dynamics of a quadratic function. This produces the characteristic period doubling sequence of the logistic map. ${ }^{22}$ In contrast, the dynamics of period- 2 iterates $T_{E}$ are not explained by similar dynamics.

Experimentally, only period-2 patterns have been observed in explosively crystallized films. ${ }^{23,24}$ This implies that it is possible to measure the points $T_{0}(2)$. However, to our knowledge no pattern of period 4 or greater has been observed in a crystallized amorphous sample. We believe the reason for this is because of the decreasing $T_{0}$ range available for bifurcations to occur, with increasing $\rho$. To see this we must first convert the $T_{0}$ intervals over which period 


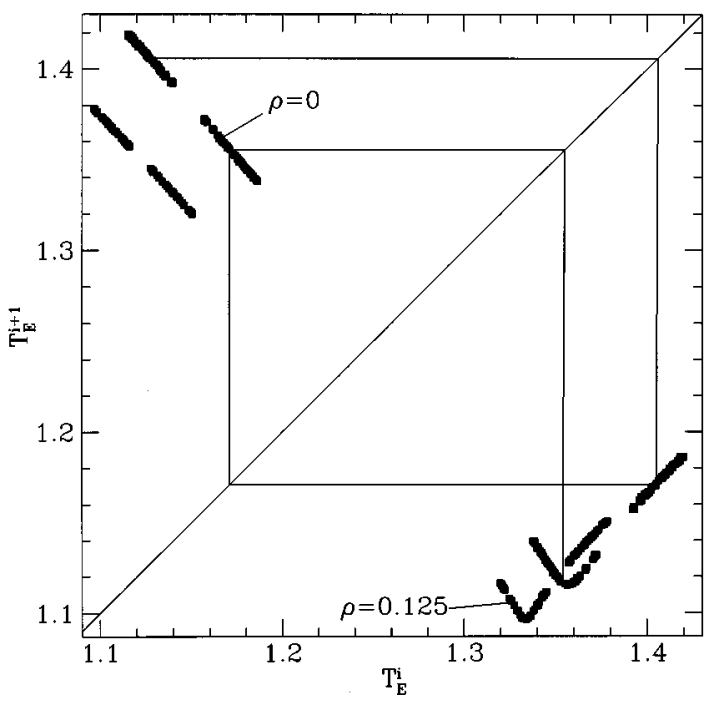

FIG. 9. Plots of $T_{E}^{i+1}$ versus $T_{E}^{i}$ for $\rho=0$ and 0.125 . A sample iteration sequence is shown for the $\rho=0$ map. These mappings have two different structures. The linear parts are responsible for overall period-2 cycling of $T_{m}$, while the smaller quadratic components are responsible for the finer structures of the bifurcation diagrams, occurring for period $2^{n}, n=2,3, \ldots$, solutions of $T_{m}$. These finer structures produce the characteristic period doubling generated by the logistic map.

doubling bifurcations occur into unscaled units of temperature. In dimensionless units, the largest interval over which period doubling bifurcations occur is $\Delta T_{0} \sim 0.005$, corresponding to $\rho=0$, as shown in Fig. 5. Using $q=300 \mathrm{~K}$ (in the range of $\mathrm{Sb}$ ), our model predicts a temperature range of $\Delta T_{\text {sub }} \sim 1.5 \mathrm{~K}$. Thus, according to our model, $\Delta T_{\text {sub }}<1.5 \mathrm{~K}$ for any finite value of $\rho$. This magnitude of temperature presents a problem, as it will often correspond to the limit of temperatures at which an amorphous film can experimentally be maintained. Any fluctuations of order $T_{\text {sub }} \sim 1 \mathrm{~K}$ would, in theory, cause $T_{E}$ to jump to different locations on the bifurcation diagram (perhaps even below the point of sustaining crystallization). It is thus unlikely that higher period doubling bifurcations can be observed in the systems undergoing an amorphous-crystalline transition for a nonzero value of $\rho$.

We also examined the dependence of $\epsilon$ on $\rho$ and $T_{0}$, numerically. Our estimates of these two parameters show that $\epsilon$ is essentially completely determined by the concentration diffusion, for the range of substrate temperatures examined. This estimate will be useful to us below. Since $\epsilon$ is oscillatory for $T_{0}<T_{0}(2)$ we considered the averaged $\epsilon$. The interfacial width is defined as $\epsilon(t)=1 / m(t)$, where $m(t)$ is the slope of the $C$ field at the point where $C(x, t)=1 / 2$. When in the oscillatory part of parameter space, the average value was obtained by averaging $1 / m(t)$ over many cycles of a periodic solution.

For the particular range of $\rho$ examined numerically, namely, $0<\rho<0.33$, and for $T_{0}$ fixed very near $T_{0}=T_{0}(2)$, we found that $\epsilon$ is well approximated by

$$
\epsilon\left(\rho, T_{0}(2)\right) \approx 0.7 \rho+3.4 .
$$

This functional form remains valid over the range $T_{0}(2)-\Delta T_{0}(\rho)<T_{0}<T_{0}(2)+\Delta T_{0}(\rho)$, where $\Delta T_{0}(\rho)$ is chosen such that $T_{0}$ never goes below the point where crystallization dies out. Even for the expanded range $T_{0}(2)-\Delta T_{0}(\rho)<T_{0}<T_{0}(2)+\Delta T_{0}(\rho=0)$, the form of $\epsilon$ remains essentially independent of $T_{0}$. Equation (34) is in accord with intuition, as concentration diffusion is expected to broaden the boundary layer of the $C$ field. Increases of $\epsilon$ with $\rho$ occur predominantly on the leading edge of the reaction front where the second derivative term in the $C$ equation is dominant.

Some of these results can be obtained by analytic arguments. Motivated by our numerical work, we make a simple decoupling ansatz for the concentration field and the reaction rate, namely,

$$
C(x, t)=c\left(\frac{x}{\epsilon}\right), \quad R(T)=R[T(0, t)] r\left(\frac{x}{\epsilon}, \rho\right),
$$

where the width of the interface $\epsilon(t)$ enters. From our numerical work, the field $c \approx 0$ for $x \leqslant 0, c \approx 1$ for $x \geqslant 2$ and $r(0) \approx 1$, while $r(x) \approx 0$ for $x>2$. Using these forms, we can integrate Eq. (5) for the concentration, thereby finding the form of the velocity and the source term in the temperature equation; namely,

$$
\frac{\partial T}{\partial t}=\frac{\partial^{2} T}{\partial x^{2}}+V(t) \frac{\partial T}{\partial x}-\left(T-T_{0}\right)-S(x, t),
$$

where

$$
S(x, t)=\left(V(t)+x \frac{\partial \ln \epsilon}{\partial t}\right) \frac{1}{\epsilon(t)} \frac{d c(z)}{d z},
$$

$z=x / \epsilon$, and

$$
V(t)=a_{0} \epsilon(t) g R[T(0, t)]-b_{0} \frac{d \epsilon(t)}{d t} .
$$

The constants $a_{0}$ and $b_{0}$ are integrals of the $c$ field: $a_{0}=c_{0} \int_{-\infty}^{\infty} r c(d c / d z) d z, \quad b_{0}=c_{0} \int_{-\infty}^{\infty} z(d c / d z)^{2} d z, \quad$ and $c_{0}=\left[\int_{-\infty}^{\infty}(d c / d z)^{2} d z\right]^{-1}$. From our numerical work, we can estimate the values of $a_{0}, b_{0}, c_{0}$, and other integrals involving $c$.

Our ansatz, then, brings our model to a tractable form, and we can proceed as in the linear stability analysis of the steady states done by van Saarloos, Weeks, and Kurtze. ${ }^{9}$ Two features of our model survive the decoupling ansatz, and differ from the earlier work. First, although we shall make no use of it here, the time dependence of the width allows for the time evolution of the temperature field to slip out of synchronization with the concentration field, which we have seen numerically for complicated doubling sequences. Secondly, since the source is not $\delta$-function-like in this limit, we can obtain the postponement effect of the period doubling due to the concentration diffusion.

In the steady-state limit, the solution of the temperature equation can be obtained easily from the Green function

$$
G(x)=-\sqrt{\tau} \exp -\left(\frac{v}{2} x+\frac{1}{2 \sqrt{\tau}}|x|\right)
$$

where the steady-state velocity is given by $v=a_{0} \epsilon g R$, and $\tau=1 /\left(v^{2}+4\right)$. The steady-state solution is formally given by 


$$
T(x)=\int d x^{\prime} G\left(x-x^{\prime}\right) S\left(x^{\prime}\right) .
$$

The linear stability analysis of time-dependent fluctuations around these solutions can now be done. This analysis is straightforward for small interfacial widths, but tedious, and is similar to that of the original work. ${ }^{9}$ Complete details are given in Ref. 15. In any case, we find that oscillations appear in the linear stability analysis corresponding to initial bifurcation of $T_{m}$. The dependence of this bifurcation on $\rho$ is

$$
T_{0}(2)=\left.T_{0}(2)\right|_{\rho=0}+a\left(1-e^{-b \rho}\right),
$$

where $a \approx 0.38$ and $b \approx 2.8$. These values are approximate because integrals such as $a_{0}, b_{0}$, and $c_{0}$ must be numerically estimated. Nevertheless, this gives us additional confidence in the result obtained from the numerical simulations reported above and in Fig. 6.

\section{SUMMARY}

We have proposed a phase-field model describing reaction-front propagation in activated transitions obeying Arrhenius chemical kinetics. We have illustrated the model by applying it to explosive crystallization in thin amorphous films. Asymptotic analysis shows that our model simplifies, in the limit of large reaction rate and small concentration diffusion, to a sharp interface model, which has been used previously. In our model, reactant concentration couples to the thermal field, altering the bifurcation properties of the propagating front predicted in the sharp interface limit. In particular, the temperature at which periodic solutions first appear depends strongly on reactant diffusion, shifting — or being postponed - to lower substrate temperatures as reactant diffusion is increased. The dependence of this temperature $T_{0}(2)$ on the reactant diffusion coefficient $\rho$ was examined and found to be well fitted to an exponential, over the range of $\rho$ examined. Analytic arguments also gave weight to this form. This dependence could be tested experimentally.

We also found that periodic solutions of $T_{m}(t)$ generated a sequence of period doubling bifurcations, for numerous values of $\rho$. A first return mapping constructed from $T_{m}(t)$ showed that for bifurcations of period 4 and higher, these bifurcation diagrams generate the same period doubling sequence of the one-dimensional logistic map. However, we found that as $\rho$ increased the substrate temperature range on which this period doubling occurs decreased. We calculated that this range spanned no more than $1 \mathrm{~K}$. It is likely that the temperature of clamped amorphous thin films cannot be controlled to this tolerance, and so we expect that higher order bifurcations are unobservable in these systems.

\section{ACKNOWLEDGMENTS}

We thank Ivan L'Heureux for useful comments. This work was supported by the Natural Sciences and Engineering Research Council of Canada, and les Fonds pour la Formation de Chercheurs et l'Aide à la Recherche de Québec. K.R.E. acknowledges the support of Grant No. NSF-DMR-8920538, administered through the University of Illinois Materials Research Laboratory.
${ }^{1}$ C. C. Coffin and S. Johnston, Proc. R. Soc. London Ser. A 146, 564 (1934).

${ }^{2}$ T. Takamori, R. Messier, and R. Roy, Appl. Phys. Lett. 20, 159 (1972).

${ }^{3}$ R. A. Lemons and M. A. Bosch, Appl. Phys. Lett. 39, 343 (1981).

${ }^{4}$ C. E. Wickersham, G. Bejor, and J. E. Green, Solid State Commun. 27, 27 (1978).

${ }^{5}$ H. J. Zeiger, J. C. C. Fan, B. J. Palm, R. L. Chapman, and R. P. Gale, Phys. Rev. B 25, 4002 (1982).

${ }^{6}$ C. S. Haase, J. Chadam, D. Feinn, and P. Ortoleva, Science 209, 272 (1980).

${ }^{7}$ I. L'Heureux, Phys. Rev. E 48, 4460 (1993).

${ }^{8}$ P. C. Hohenberg, Phys. Scr. T9, 93 (1985).

${ }^{9}$ W. Van Saarloos and J. D. Weeks, Phys. Rev. Lett. 511046 (1983); Physica D 12, 279 (1984); D. A. Kurtze, W. Van Saarloos, and J. D. Weeks, Phys. Rev. B 30, 1398 (1984).

${ }^{10}$ R. Kapral, J. Math. Chem. 6, 113 (1991).

${ }^{11}$ W. Lotmar, Helv. Phys. Acta 18, 369 (1945).

${ }^{12}$ A. Gotzberger, Z. Phys. 142, 182 (1955).

${ }^{13}$ O. Bostanjoglo and G. Schlotzhauer, Phys. Status Solidi A 68, 555 (1981).

${ }^{14}$ V. M. Kuzmenko and V. I. Melnikov, Zh. Éksp. Teor. Fiz. 82, 802 (1982) [Sov. Phys. JETP 55, 474 (1982)].

${ }^{15}$ N. Provatas, Ph.D. thesis, McGill University, 1994.
${ }^{16}$ R. F. Sekerka, in Proceedings of the International School of Crystallography, Erice, 1981, edited by R. F. Sekerka (Reidel, Dordrecht, 1982).

${ }^{17}$ M. E. Fine, Phase Transitions in Condensed Systems (MacMillan, New York, 1964).

${ }^{18}$ G. H. Gilmer and H. J. Leamy, in Laser and Electron Beam Processing of Materials, edited by C. W. White and P. S. Peercy (Academic, New York, 1980), p. 227.

${ }^{19}$ A. K. Kapila, in Asymptotic Treatment of Chemically Reacting Systems, edited by A. Jeffrey (Pitman Advanced Publishing Program, Boston, 1983).

${ }^{20}$ A. H. Nayfeh, Introduction to Perturbation Techniques (John Wiley \& Sons, New York, 1981).

${ }^{21}$ G. Caginalp, Phys. Rev. A 39, 5887 (1989).

${ }^{22}$ M. Feigenbaum, Los Alamos Sci. 1, 4 (1980). This paper is reproduced in the Proceedings of the International Conference on Order in Chaos [Physica D 7 (1983)].

${ }^{23}$ D. J. Wollkind, in Preparation and Properties of Solid State Materials, edited by W. R. Wilcox (Marcel Dekker, New York, 1979), Vol. 4.

${ }^{24}$ K. Kerrihara, K. Sasaki, and M. Kawarada, in Proceedings of 1st International Symposium on Functionally Gradient Materials, edited by M. Yamanouchi, M. Koizumi, T. Hirai, and I. Shiota (Functionally Gradient Materials Forum, Sendai, Japan, 1990). 